\title{
De l'objet de désir aux sujets désirants
}

\author{
Le féminin sacré et la mère nature
}

Ghaliya Djelloul

Delphine Masset

\begin{abstract}
[Résumé] Dans le cadre de références mobilisé par les écologistes, le mot « nature » est souvent convoqué. Celui-ci, de par sa polysémie, recouvre cependant une large palette de réalités. II a acquis, au gré des constructions sociales et historiques, des significations diverses.

Le mot «femme » renvoie, lui aussi, à un imaginaire pluriel et protéiforme. Dans cet imaginaire, cette « créature » a de nombreuses fois été associée à la nature. Il y aurait en effet une essence féminine caractéristique ; celle-ci l'amènerait à incarner la mèreterre, l'environnement. La femme se vivrait donc, pour certains, dans un rapport d'opposition à la culture. Mais quel est ce « naturel » féminin et qui plus est, s'il induit vraiment une quelconque forme de «nature », quels en sont ses présupposés?

Dans cet article, nous étudierons les différentes significations qui associent femme et nature (1). Ensuite, nous essayerons de relever les écueils propres à cette association (2). Le mobile sous-tendu par cet article étant d'éviter aux femmes de s'associer, parce qu'elles seraient écologistes, à des significations réductrices et décapacitantes de la nature. Notre postulat est en effet que les images mobilisées vis-à-vis de la nature et des femmes relèvent d'une vision du monde et des rapports de force genrés, autrement dit d'un « ordre social des sexes ». En effet, nous verrons comment l'essentialisation des femmes, par l'enfermement dans un type d'être inadéquat, les propulse en dehors de la sphère de la polis.

Nous souhaitons mettre en lumière cette série d'associations et développer les incidences qu'elles peuvent avoir en matière de constructions identitaires de la féminité. Notre réflexion portera donc sur les représentations de la « féminité » relatives à la nature.
\end{abstract}

Mots-clés : féminité, nature, engagement.

\section{La construction historique du rapprochement des femmes à la nature}

Les tentatives d'identifier « femme » et " nature » dans l'héritage judéo-chrétien sont présentes dès les mythes fondateurs. En effet, « L'expulsion de l'Eden et la chute originelle ne sont-elles pas le résultat d'un pacte entre la femme et la nature incarnée par le 
serpent, qui oppose au jardin bien ordonné le chaos et la barbarie d'un monde dé$\mathrm{chu}^{1}$ ? ». On voit par ailleurs que dans cet héritage (et comme l'a décrit Simone de Beauvoir), la femme incarne la figure de l'altérité, le contraire ou le non-homme, tout comme la " nature » incarne une altérité radicale par rapport à la « culture » : « [La nature] peut être vierge ou fertile, elle doit être ensemencée pour donner ses fruits, elle doit être domptée et tenue en place pour ne pas menacer l'ordre social. La féminité comme la nature sont donc considérées comme l'Autre, le chaos, le contraire de l'ordre et de la rationalité qui s'incarneraient dans les vertus masculines ${ }^{2} »$.

Or, la femme n'a pas toujours été considérée comme l'Autre de l'homme. Que ce soit pour les Grecs anciens ou pour les Japonais traditionnels, «le problème n'était pas d'avoir une relation amoureuse ou sexuelle avec un autre homme (...) mais que soit respectée la hiérarchie de rang entre le partenaire actif et le partenaire passif ${ }^{3}$ ». Il existe donc d'autres structures/systèmes que celles du genre pour ordonner le monde, comme il existe d'autres manières d'appréhender celui-ci qu'au travers de la distinction natureculture.

Quoi qu'il en soit, l'analogie occidentale entre le clivage femme-homme et natureculture dont nous avons hérité n'est pas sans conséquence pour la construction identitaire des sujets dits « féminins ». La nature étant pensée comme limitante et la culture comme capacitante, ces distinctions configurent les sphères d'existence et les types d'activités liées à chaque genre, et déterminent, in fine, l'appropriation des lieux de pouvoir propres à la sphère de la culture.

\section{La « nature » immorale de la femme}

Si la corrélation faite entre femme et nature propre à l'héritage judéo-chrétien renvoie au chaos, comme le suggère Virginie Maris, ou encore à une dimension instinctive qui devrait être domptée, qu'elles en sont les conséquences?

Cette mythologie, répercutée au travers d'un processus de domination sociale pluriséculaire, a en effet des incidences sur les schèmes mentaux de construction de la psyché et de l'identité féminine.

Nous proposons de passer en revue deux constructions sociales d'archétypes de " femmes » qui renvoient, d'une manière ou d'une autre, à ce réservoir d'images et de symboles qui associe la femme aux chaos et incite celle-ci à compenser le maléfique de sa nature féminine par une vertu irréprochable. Ces deux archétypes se présentent

\footnotetext{
${ }^{1}$ Maris V., citant Carolyn Merchant dans « Reinventing Eden », Virginie Maris « Quelques pistes pour un dialogue fécond entre féminisme et écologie », Multitudes 1/2009 (n³6), p. 178-184

${ }^{2}$ Maris V., « Quelques pistes pour un dialogue fécond entre féminisme et écologie », Multitudes 1/2009 (n³6), p. 178-184.

${ }^{3}$ Macé É., « Le piège de la « cause des femmes » » (troisième partie), 14 nov.2003.
} 
comme deux types d'économie psychique qui visent à résoudre le péché originel, par l'exacerbation de la bienveillance ou le refoulement de ses vices présumés.

La chasse aux sorcières, qui a principalement eu lieu entre 1580 et 1630, serait à ce titre emblématique d'une représentation des femmes (et de sa résolution historique) comme êtres maléfiques. Patrick Snyder parlera lui d'un " phénomène de la chasse aux sorcières comme le procès de 'l'être-pour-le-mal't.»

L'image de la femme à travers le préromantisme et le romantisme témoigne, elle, d'un autre cliché : la femme angélique, de préférence sœur, vierge ou morte ${ }^{5}$. Ainsi, la femme comme la « nature » deviendra au cours du romantisme, et par compensation, synonyme de bienveillance et de pureté. On voit que : « dans le courant romantique et plus encore chez les préservationnistes américains, on vénère une nature pure et vierge, qui seule permet de s'élever au-delà de la culture jugée mercantile et décadente [mais que] loin d'améliorer la perception générale des femmes, l'image de la Vierge écrase toutes celles qui ne le sont pas, elle réduit l'ensemble des femmes profanes, donc toutes les femmes réelles, à la banalité et à l'imperfection ${ }^{6}$.»

Ainsi, la nature et par corrélation la femme sont investies de valeurs contradictoires? Ève la pècheresse et Marie la vierge et la sublime renvoient à des imaginaires opposés, bien que toujours idéalisés. Cependant, qu'elles soient une icône ou une menace, la femme, comme la nature, renvoie bien à cet Autre mystérieux et irréductible, et donc, objet de projection. Ainsi «concentrer son attention sur la nature sauvage et grandiose incite à délaisser la nature dite ordinaire, celle dans laquelle et avec laquelle on vit tous les jours 8 ", tout comme faire de " la femme » un idéal imaginaire de "sainte » nous éloigne de qui sont réellement les femmes dans leur multiplicité et complexité.

Ainsi, si les chaînes qui empêchaient les femmes de s'émanciper se sont desserrées, les femmes restent encadrées et modelées par une série d'archétypes qui se sont construits autour de l'héritage judéo-chrétien. Il semblerait qu'aujourd'hui encore, certains réflexes féminins contemporains fassent échos à des économies psychiques basées sur la résolution de ce péché originel. Ces économies psychiques proposeraient, pour combler l'image coupable et entachée de la nature féminine, d'incarner la Vierge ou la vertu, une moralité irréprochable.

LA VERTU ET LE VOILE. Une des tentatives d'incarner cette supériorité morale est sous-tendue par le port du voile dans la tradition musulmane. Il y aurait beaucoup d'éléments de contextualisation à avancer pour montrer la polysémie du port de cet habit/accessoire par les femmes selon les contextes sociohistoriques (Göle, 2003), mais, in fine, il renvoie à une exhortation à la pudeur, qui se décline différemment selon le

\footnotetext{
${ }^{4}$ Snyder P. ; Représentations de la femme et chasse aux sorcières, XIIIle-XVe siècle, Préface du livre par Michel Dion.

${ }^{5} \mathrm{http}: / /$ www.lettres.ac-versailles.fr/spip.php?article319

${ }^{6}$ Maris V., « Quelques pistes pour un dialogue fécond entre féminisme et écologie », Multitudes 1/2009 (n 36), p. 178-184.

${ }^{7} \mathrm{Idem}$.

${ }^{8}$ Idem.
} 
genre. L'invitation à la pudeur et à ne pas faire usage de ses charmes est justifiée par le maintien de l'ordre social et la peur de la fitna, c'est-à-dire de toute tentation qui écarterait du chemin de la piété. Ce terme renvoie en arabe aussi bien à la beauté "fatale " d'une femme, qui perturbe l'homme croyant, qu'aux conflits entre communautés et au danger de « guerre civile » (Mernissi 1992). Dans un cadre discursif islamique, les valeurs de « pudeur » et de « chasteté » sont des éléments-clés d’une religiosité genrée.

\section{Archétypes féminins}

Nous allons maintenant passer en revue deux archétypes féminins présents dans le contexte occidental et la tradition judéo-chrétienne : celui de «la sainte » et de « la belleâme ». Nous montrerons par ceux-ci comment le rapprochement entre femme et nature peut parfois se décliner.

\subsection{L'archétype de la sainte}

Comme l'expliquait Virginie Maris dans l'extrait précédent pour la Vierge, l'amalgame propre aux romantiques est celui d'une nature généreuse et sans limites, et d'une femme renvoyant aux mêmes caractéristiques. Pour les romantiques, la nature renferme le privilège d'être pure, entière et éclatante : elle n'est entachée d'aucun vice et ne connaît ni souillure ni corruption. Par analogie, la sainte incarnera un modèle de vertu, ne portant en elle que de bons sentiments, des inclinations à l'amour, pures et lumineuses. Elle sera, elle aussi, " généreuse et sans limites ».

Cette figure induit une exacerbation de la sensibilité qui repose sur une logique d'amour et de compassion. La Sainte est en effet aussi bien une figure historique issue du romantisme que du piétisme ${ }^{9}$. Dans le piétisme, l'émotion joue un rôle prépondérant : est réputé élu du « Divin » l’individu, qui, par ses capacités imaginatives, parvient à ressentir la pitié du « Père " pour le monde. Le romantisme, esprit hybride qui prend racine dans le piétisme, mettra l'accent sur un rapport sincère, authentique et non ostensible de cette sensibilité ${ }^{10}$. Il s'agira de consacrer sa vie à aimer Dieu et les hommes.

Cette prescription à l'amour et l'incarnation d'une nature douce, passive et réconfortante n'est pas sans lien avec une conception idéalisée de la nature. L' « alma mater » et la nature dépeinte par les romantiques ne sont-elles en effet source de réconfort et de contemplation?

\footnotetext{
${ }^{9}$ Doctrine qui se développe dans le giron de Luther, le piétisme est un mouvement spirituel qui se développe au 17e siècle.

${ }^{10}$ Arnsperger et Périlleux, Syllabus, Cours 7, p. 5.
} 


\subsection{L'archétype de la belle-âme}

La deuxième figure qui rapproche femme et nature est celle de la belle-âme. Est naturel, dans ce cas, ce qui est conçu comme « spontané ». Elle ferait échos à une femme en recherche d'authenticité. L'essentialisme féminin y recouvre la figure de la « belleâme » schillérienne ${ }^{11}$ pour laquelle il faudrait n'avoir aucune responsabilité avilissante ou salissante. Plutôt que de se vivre en retrait (dans une forme d'extraction du monde) et de refouler ses désirs inadéquats comme l'aurait fait l'archétype de « la sainte », « la belle-âme » fait correspondre sa « nature sensible » à la loi morale, à l'instar de la « belle âme » de Schiller qui unissait l'instinct et le devoir. En ce sens, l'incorporation du devoir est profond : il est l'indice d'un pouvoir tentaculaire, qui va jusqu'à s'insinuer dans l’» âme ». Pour « la belle-âme », il s'agit d'incorporer la loi morale pour pouvoir continuer à être libre. Autrement dit, sa sensibilité l'amène à avoir des émotions et des comportements qui sont attendus d'elle. Schiller décrit:

« la belle-âme » en ces termes : elle « ne pourra conserver la faculté de choisir et cependant être un chaînon sûr dans la chaîne causale des forces que si dans le monde des apparences, les deux mobiles de l'inclination et du devoir engendrent des effets complètement identiques et que si, quelle que soit la forme de la volonté, la matière en demeure la même : il faut donc que ses instincts se trouvent avec sa raison en un accord tel qu'ils soient mûrs pour une législation universelle ${ }^{12} »$.

Ce régime d'être incarne une nature spontanée, authentique, en deçà des modes de socialisation. Elle n'est, évidemment, qu'un artifice et une illusion rendue possible par l'incorporation de la norme. Loin d'envisager d'avoir des comportements stratégiques ou de s'essayer à un arbitrage rationnel quant à la nécessité de maintenir certains devoirs pour « faire société », « la belle-âme » préférera s’imaginer libre et s'engager dans le monde de ses émotions et de sa subjectivité.

\section{L'arbitrage nature-culture ou une décapacitation organisée}

Comme présenté en amont, le clivage/dichotomie homme-femme ferait échos au clivage culture-nature. Reviendrait alors à la femme le monde de la nature et ses corollaires, notamment le souci naturel du bien-être des autres, et à l'homme, la culture qui se décline matériellement en un ensemble d'instances de socialisation avec, comme emblème, la loi.

Ainsi, la nature serait dans l'émotion, l'intuition, la spontanéité des intentions tandis que la culture consacrerait le jeu social, les agencements stratégiques, l'image publique et le jugement. La nature veut rassembler et unifier alors que la culture dissocie (les rôles, les fonctions), sépare (les obéissants des désobéissants) et instaure les hiérarchies

\footnotetext{
${ }^{11}$ Schiller, Lettres sur r'éducation esthétique de l'homme, trad. Robert Leroux, Paris, Aubier, 1992.

${ }^{12}$ Idem, Quatrième Lettre, p. 103.
} 
(classes, générations). Or, c'est bien toujours la culture qui domine la nature, rapport fondamental à partir duquel la distribution sociale s'opère.

Cette distinction, si elle est opérante, implique deux choses pour les femmes qui tendraient à s'associer à la nature au travers des figures archétypiques précédemment présentées : la mise en péril du « soi » qui les éloigne de l'enjeu du droit à faire loi (A) et les incline à la passivité (B). Regardons de plus près les risques qu'encoure la femme. Passons en revue ces deux écueils.

\section{L'aveuglement aux bonnes intentions et l'interdiction de faire loi}

Il y a chez cette figure de femme archétypale un aveuglement, parfois entretenu, à ne pas percevoir les dangers. Cet aveuglement permet d'éviter de réagir brutalement, en niant la violence ou en la retournant contre soi. Cette violence peut en effet être retournée : le culte de l'amour et de la sensibilité chez la Sainte peut déboucher sur un désir sacrificiel, un désir de martyre. C'est ce qu'illustre par exemple l'extrait d'une lettre adressée et reprise par Simone de Beauvoir: « J'aurais voulu être une sainte de l'amour. J'enviais le martyre dans de tels moments d'exaltation et de fureur ascétique ${ }^{13}$. » Un autre exemple de cet amour inconditionnel qui mène à une subordination brutale est le roman Une vie de Guy de Maupassant (1883). L'héroïne y exprime parfaitement la noblesse de sa nature, à savoir celle de ne pas être touchée par les bassesses des autres et de maintenir le feu sacré de l'amour dans l'abandon total, en subissant les maltraitances et les abus de son mari, puis de son fils. En montrant une ignorance totale de sentiments de colère, de haine, de revanche, elle est un brillant exemple de la vie vertueuse qu'une femme de son rang et se son époque était censée avoir.

L'enjeu de cette « vertu féminine » est donc d'entretenir une naïveté et une innocence au fondement de la capacité à "se donner ${ }^{14}$ ", plutôt que d'incarner " une tête bien faite ", maline et astucieuse et dont l'intérêt d'un rapport stratégique aux choses n'échappera pas.

Ce type d'économie psychique, dont la visée ultime est l'amour (ou l'image de l'amour), organisera ainsi sa propre soumission. Celle-ci sera préférée à celles des autres ou jugée comme une contrepartie tenable à la participation au monde des hommes. Ainsi, dans un mode d'être où l'amour est sanctifié, le sacrifice sera bien souvent banalisé : « Pour réaliser cette union, ce que la femme souhaite d'abord, c'est servir ; c'est en répondant aux exigences de l'amant qu'elle se sentira nécessaire, elle sera intégrée à son existence à lui ${ }^{15}$. »

${ }^{13}$ de Beauvoir S., Le deuxième sexe II, Gallimard, 1976, p.549.

${ }^{14}$ Françoise Collin ira jusqu'à parler d'une assignation des femmes au don et de la violence qu'elles subissent en guise, semble-t-il, de version perverse du « contre-don ». COLLIN F., « Donner par « nature », est-ce donner? », Cahiers du Genre, n53/2012

${ }^{15}$ de Beauvoir S., Le deuxième sexe ll, Paris : Gallimard, 1976, p.550. 
L'amalgame, propre au romantisme, qui vise à associer femme, nature et douceur est foncièrement décapacitant. Celui-ci n’a pas sorti les femmes de leurs conditions : « les transports des poètes n'avaient aucun effet sur la situation légale ou économique des femmes et n'influaient gère sur leur statut social. (...) L'amour romantique masque les réalités de la situation féminine et le fardeau de la dépendance économique ${ }^{16}$.

La vulnérabilité de ces figures idéalisées tiendrait donc en une volonté de pureté qui rendrait aveugle à la violence et donc, inapte à s'en servir en cas de besoin ; ainsi qu'à une morale poussant à respecter la « nature » des choses et rendant peu vigilantes.

\section{L'ascétisme et la passivité comme dénominateur commun}

En rapprochant nature et culture chez la femme, les pulsions et l'agressivité deviennent une sorte de résidu gênant pour la civilisation et les hommes. Celui-ci doit être évacué, soit sous forme de refoulement (en rejetant, de fait, tout comportement répressif, prédateur, etc.), soit sous forme d'incorporation de la norme. L'effet de ce refoulement ou de cette incorporation ne serait pas très éloigné d'une vie faite d'affects passifs, en référence à la terminologie spinoziste ${ }^{17}$. La manière de rentrer en relation serait alors caractérisée par une passivité totale et entretenue face aux évènements. Cette passivité relève d'un décapacitement de " la femme » à être maîtresse de son destin social, elle la confine à des rôles et des espaces définis. « La sainte » ou « la belle-âme » auraient tendance à ne pas « composer avec les choses ». Ces femmes pourraient développer des désirs d'ascétisme en abordant l'existence avec un refus de vivre dans le « rapport des choses »- de s'opposer, de revendiquer, de crier, de forcer, etc. Ainsi, dénuée de «bon sens » quant aux réelles menaces que le monde pourrait présenter pour elle, elle s'engagerait, tête baissée et avec un dévouement complet, dans des actions ou des relations qui pourraient l'affecter négativement. C'est donc une partie de l'agencéité (Montenach 2012), ou capacité sociale d'agir qui est retirée aux figures archétypales de « femme vertueuse par nature».

\section{Une posture morale qui arrive à compenser ces décapacitations ?}

Aux postures de "sainte » ou de " belle-âme » se greffe une morale compassionnelle dont les caractéristiques sont le respect pour l'inclination naturelle des êtres, une compréhension pour les comportements « naturels » et instinctifs. L'attachement à la nature (entendre « instinct») agit ici comme morale. L'instance de socialisation (la « culture ») que sont la loi et la répression auront tendance à être refusées (jugées trop

\footnotetext{
${ }^{16}$ Millett K., La politique du mâle, Paris : Stock, 1971.

${ }^{17}$ Voir le document audio : «In memoriam Gilles Deleuze ».
} 
brutales et injustement excluantes), au bénéfice d'une morale " compatissante », qui se trouve parfois confrontée à ses propres limites.

Le film « Manderlay » de Lars Von Trier, met en scène cette femme archétypique. On y voit une femme (qui s'appelle de manière significative " Grace ») qui se sacralise en adoptant une posture morale proche de la sainteté. La scène finale du film confronte le père et sa fille, après qu'une communauté de villageois l'ait accueillie mais aussi exploitée et abusée, sans qu'elle n'y apporte aucune résistance.

«-Grace : Ce n'est pas moi qui juge, c'est toi.

- Son père : Toi tu ne juges pas parce que tu compatis - une enfance malheureusement et un meurtre n'est plus un meurtre, n'est-ce pas ?

- Grace : Tu blâmes seulement les circonstances.

_Son père : Les violeurs et les criminels sont des victimes selon toi, moi j'appelle ça des chiens, et s'ils bouffent leur propre vomi, on doit les fouetter.

_Grace : Mais un chien n'obéit qu'à sa propre nature alors pourquoi ne pourraiton pas lui pardonner ça?

- Son père : On ne pourrait rien apprendre au chien si on leur pardonnait tout le temps lorsqu'ils obéissent à leur propre instinct.

- Grace : Donc je suis arrogante, je suis arrogante parce que je pardonne les gens?

- Son père : Te rends-tu compte à quel point tu es condescendante ? Tu es persuadée à l'avance que personne ne peut atteindre ton niveau de moralité. Alors tu excuses. Je ne vois rien de plus arrogant. Tu pardonnes aux autres ce que tu ne pardonnerais jamais à toi-même. »

Alors que l'héroïne bâtit sa morale autour de l'amour du prochain (malgré tous les sévices que ce prochain peut lui faire subir), son père érige la sienne à partir d'un désir de " civilisation » : il propose une morale conséquentialiste. Celui-ci blâme le comportement des individus en raison de leurs conséquences négatives. La morale de l'héroïne est ainsi construite que les actes les plus vils ne sont pas réprimandables (voire réprimés) en raison de leur origine instinctuelle. Cette morale omet une prise en compte des conséquences, afin de maintenir ce qui l'a fondé : une volonté de ne pas nuire. Cette morale a l'avantage d'esquiver une série de nécessités, dont celle de recourir à la violence dans le cas d'une agression.

C'est Virginie Despentes qui rappellera, avec sa très controversée " King Kong Theory ", que la femme, elle aussi, peut avoir besoin d'utiliser la violence et qu'elle en est décapacitée par un manque d'apprentissage de l'agressivité. Le corps de «la femme » est investi d'une moralité qui nie toute possibilité de s'engager, en tant que sujet, dans une lutte à armes égales. Elle doit, pour réaliser son « penchant » naturel, être plus vertueuse et plus noble que le commun des mortels.

\section{Coûts et bénéfices moraux de la décapacitation}

Si ces descriptions peuvent sembler caricaturales et ces postures ingrates à entretenir, il ne faut pas négliger le fait qu'elles sont soutenues par la création d'une valeur : celle de la valeur morale (constituant un capital vertueux) de celle qui l'adopte. La « sainte » 
ou la « belle-âme » sera tenue par un sentiment de fierté, dû à l'existence, la conservation et le perfectionnement de sa vertu. Si on peut lui reprocher de ne pas s'impliquer dans le monde, elle pourra toujours rétorquer d'au moins de pas être à son image. Plus morale et vertueuse, ces archétypes féminins penseront avoir les privilèges de leur défaut.

Ainsi, si ces postures peuvent sembler décapacitantes (refoulement de ses désirs propres, retrait, faible emprise sur le monde, susceptibilité et vulnérabilité aux agressions), elles seront compensées par l'image d'une moralité irréprochable. Cette moralité, ce sera le ciment des identités de ces femmes archétypiques. Leur être sera soustendu par cet horizon.

\section{Conclusion : nature et féminité revisitées et politisées}

Certaines femmes se sentent plus proches d'un état de nature que de culture. Effectivement historiquement plus éloignées des sphères du pouvoir et de la " polis », certaines trouveront en la nature des sources d'identifications. Comme l'explique cette géographe «écoféministe» :

«En tant que jeune fille blanche de la classe ouvrière, je ne pouvais m'identifier au héro du Western, au rancher ou à mon voisin chasseur. Je m'identifiais au loup, au bétail, aux Indiens [Native Americans], et autres marginaux [Outsiders]. (...) C'est peut-être pour cela que je suis devenue une environnementaliste radicale $(\ldots)^{18} . »$

Si dans les exemples précédents, la nature était clairement le lieu du non-politique, l'écologie politique, elle, a permis de mettre au jour le rapport de force présent entre la culture et la nature, et la nécessité de penser la nature comme un bien commun dont découle une responsabilité collective. Ce mouvement n’a pas manqué de colorer de lunettes féministes comme l'a démontré l'écoféminisme qui valorise la féminité de la nature comme un outil de résistance à un système androcentré. Ici, les rapprochements proposés entre femmes et nature sont capacitants : être femme, et proche de sa nature, incarne un levier de résistance, de protestation symbolique et cognitive, bien que ce symbole reste construit en antithèse à la masculinité et continue d'incarner l'Autre absolu.

Or, au niveau identitaire, comment puiser des significations alternatives dans ce rapport entre genre et nature, comment dépasser ou subvertir le rapport de force ? On peut garder l'idée d'une « nature » si elle est capacitante, mais remettre en cause toute idée de vertu ou on peut remettre en cause l'idée de nature elle-même. Le problème avec cette seconde option c'est qu'elle n'offre pas d'alternative identificatoire qui permette un ancrage subjectif de « sujet ». Comment les femmes peuvent-elles donc se penser en

\footnotetext{
${ }^{18}$ Zitouni B., « L'écologie urbaine: mode d'existence? Mode de revendication? » In : Co s mo politiques, 7 août 2004, p9. Citation reprise de Wolch \& Emel, 1998, p.111-112.
} 
« sujets » alors que la catégorie même de femmes est marquée du sceau de l'altérité et non de l'intégralité (ou auto-engendrement identitaire)?

En effet, les dangers de la « belle-âme » ou de la « sainte » est de ne pas pouvoir penser son identité, sa personne, de manière plurielle. Celle-ci est réduite à l'unique expression d'une nature sensible, de manière soi-disant spontanée et authentique (ce qui n'est pourtant pas évident à la lumière de toutes les compromissions inconscientes ou multiples refoulements qui devront être réalisés pour vivre en société et se cacher à soimême ses « désirs inadéquats »). Ainsi, « la femme » ne pourra pas considérer la multiplicité des rôles sociaux ou des scènes de la vie sociale comme des opportunités à se découvrir autrement ou à jouer avec sa " nature " $\operatorname{propre}^{19}$. En effet, « la belle-âme " définirait les fins à partir des moyens : les moyens seraient réduits à la sensibilité, à l'inclination... Ils ne devraient pas être adaptés aux fins. Ces archétypes féminins laisseraient donc aux hommes la capacité d'agir et de réagir mais aussi de jongler avec les figures sociales, d'engager un rapport stratégique aux choses (d'anticiper, de calculer), ou, autrement dit, d'adapter les moyens aux fins.

Cette essentialisation permanente qu'est la féminité, et son utilisation stratégique dans le cadre de l'écoféminisme nous renvoie à la difficulté de penser les identités genrées de manière hybride et plurielle ou, autrement dit, de remettre en cause l'idée homogène de nature. En effet, même si certaines femmes adhèrent à l'idée d'une "nature féminine », sont-elles pour autant vouées aux sacrifices, à chercher à coller aux images de douceur, de soin dans lesquelles elles baignent ? Ne serait-il pas à chaque personne se disant « femme » d'exprimer ce qui fait sens pour elle dans cette identité collective et politique ? Doit-on absolument renoncer à « sa féminité » ou est-ce plutôt les cadres du féminin (et symétriquement du masculin) qui doivent être élargis pour repenser le concept de nature d'un point de vue politique ? Si l'on collectivise le concept de «nature » alors on en fait le lieu fondateur de la morale, c'est-à-dire une scène inclusive, participative et capacitante pour chacune. En effet, la nature comme principe de justification des rapports de force a pendant longtemps été la seule manière d'appréhender les êtres sociaux dominés réduits et essentialisés à cette nature, tandis que le privilège des dominants était justement de pouvoir arborer des identités multiples, complexes et non réductibles. En somme de définir l'ordre «naturel » des choses.

Or, que faut-il aux femmes pour accéder à ce privilège ? La reconnaissance. Car l'identité est une construction relationnelle et être quelque chose veut avant tout dire être reconnu comme tel. C'est pourquoi la reconnaissance de l'autonomie identitaire permettrait de produire des ressources identitaires collectives et de composer des identités plurielles, de «femmes » et de plein d'autres choses encore ${ }^{20}$.

\footnotetext{
${ }^{19}$ Elle ne pourra les considérer que comme des moments de trahisons vis-à-vis d'elle-même, des expériences « contre-natures ». Tous le foisonnement de ses êtres de culture seront vus et vécus comme de monstrueuses excroissances à sa personne.

${ }^{20}$ Pour creuser la question des identités plurielles des femmes, voir, entre autre, Haraway et son Manifeste Cyborg.
} 


\section{Bibliographie}

Arnsperger C. et Périlleux T., Syllabus, p. 5.

de Beauvoir S. (1976), Le deuxième sexe II, Paris : Gallimard.

Collin F. (2012), « Donner par « nature », est-ce donner ? », Cahiers du Genre, n53.

Gole N. (2003), Musulmanes et modernes, Paris : La Découverte.

Macé É. (2003), « Le piège de la 'cause des femmes’ » (troisième partie), 14 nov. 2003.

Maris V. (2009), "Quelques pistes pour un dialogue fécond entre féminisme et écologie », Multitudes, 1/2009 ( $\left.\mathrm{n}^{\circ} 36\right)$, p. 178-184.

Mernissi F. (1987), Le Prophète et les femmes. Paris : Albin Michel.

Millett K. (1971), La politique du mâle, Paris : Stock.

Montenach A. (2012), « Agency : un concept opératoire dans les études de genre ? », Rives méditerranéennes, 41.

Snyder P. (2000), Représentations de la femme et chasse aux sorcières, XIIIe-XVe siècle, Paris : Édition Fides.

Schiller, F. (1992) Lettres sur l'éducation esthétique de l'homme, trad. Robert Leroux, Paris : Aubier.

Zitouni B. (2004), « L'écologie urbaine: mode d'existence? Mode de revendi-cation? » In Cosmopolitiques, 7 août 2004. 